Recepción: 20 / 04 / 2017

Aceptación: 20 / 05 / 2017

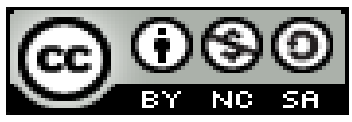

Ciencias Químicas

Publicación: 01 / 07 / 2017

Artículo Científico

\title{
Sistemas de gestión de calidad aplicada a áreas de química clínica y hematología:
} ISO 9001:2015

Quality management systems applied to areas of clinical chemistry and hematology: ISO 9001: 2015

Sistemas de gestão da qualidade aplicados a áreas de química clínica e hematologia: ISO 9001: 2015

Julio V. Rodriguez-Zurita ${ }^{\mathrm{I}}$

julio.rodriguezz@ug.edu.ec

Yuryee J. Estéfano-Limones II yuyujessi@gmail.com

\author{
Laura L. Valdez-López III \\ laura.valdezl@ug,edu.ec \\ Nilda R. Cedeño-Albán IV \\ laura.valdezl@ug,edu.ec
}

Patricia E. Rodriguez-Estéfano V pattyrodriguezestefano@gmail.com

Correspondencia: julio.rodriguezz@ug.edu.ec

\footnotetext{
I Docente de la Universidad de Guayaquil, Guayaquil, Ecuador.

II Docente de la Universidad de Guayaquil, Guayaquil, Ecuador.

III Docente de la Universidad de Guayaquil, Guayaquil, Ecuador.

IV Docente de la Universidad de Guayaquil, Guayaquil, Ecuador.

v Docente de la Universidad de Guayaquil, Guayaquil, Ecuador.
} 


\section{Resumen}

Objetivo: Diseñar una propuesta de un Sistema de Gestión de Calidad en Laboratorios de nivel II, en las áreas de química clínica y hematología, en base a los requisitos de la norma ISO 9001:2015. Metodología: La investigación que se aplicó al presente trabajo se considera de tipo descriptivo y observacional, que consistió en la recopilación de toda la información generada en las áreas de química clínica y hematología de los Laboratorios estudiados en la ciudad de Guayaquil, que generó los datos para poder diseñar la propuesta del Sistema de Gestión de Calidad en base a la Norma ISO 9001:2015 y permitió desarrollar instrumentos y técnicas que se utilizó como material de apoyo proveniente de fuentes primarias y secundarias. Resultados: Nivel A: Documento de mayor nivel en el sistema de gestión de la calidad y constituye el manual de calidad, en el que está contenida la política y objetivos de calidad y de él se derivan los siguientes niveles de la documentación. Nivel B: Lo integran los procedimientos documentados del SGC, que aplica el personal que participa en su ejecución. Nivel C: Se constituye por los procedimientos de uso general, procedimientos específicos de procesos que rigen su ejecución, procedimientos operativos o de trabajo particular de casa etapa de proceso, instrucciones de trabajo que contienen en forma detallada tareas para realizar una actividad específica (instructivos, formatos, normas). Conclusiones: Se logró ejecutar el diseño de una propuesta de un plan de implementación de la norma ISO 9001:2015 en los Laboratorios Clínicos en las áreas de química clínica y hematología, al tener un nivel de cumplimiento bajo de los requisitos de la norma en las áreas mencionadas se cuenta con un personal y directivos comprometidos es posible aplicarlo.

Palabras claves: ISO 9001:2015; gestión de calidad; laboratorios clínicos. 


\begin{abstract}
Objective: To design a proposal for a Quality Management System in Level II laboratories, in the areas of clinical chemistry and hematology, based on the requirements of ISO 9001: 2015. Methodology: The research applied to the present work is considered descriptive and observational, which consisted in the compilation of all the information generated in the areas of clinical chemistry and hematology of the Laboratories studied in the city of Guayaquil, which generated the data to be able to design the proposal of the Quality Management System based on ISO 9001: 2015 and allowed to develop instruments and techniques that were used as support material from primary and secondary sources. Results: Level A: Highest level document in the quality management system and constitutes the quality manual, which contains the policy and quality objectives and from which the following levels of documentation are derived. Level B: It is made up of the documented procedures of the QMS, which is applied by the personnel involved in its execution. Level C: It is constituted by the procedures of general use, specific procedures of processes that govern their execution, operative procedures or of particular work of house stage of process, work instructions that contain in detailed tasks to carry out a specific activity (instructive, formats, standards). Conclusions: It was possible to execute the design of a proposal for an implementation plan of the ISO 9001: 2015 standard in the Clinical Laboratories in the areas of clinical chemistry and hematology, having a level of compliance under the requirements of the standard in the These areas have a staff and committed managers is possible to apply.
\end{abstract}

Key words: ISO 9001: 2015; quality management; clinical laboratories. 


\section{Introducción.}

La información generada por los laboratorios en muchos casos es incompleta y no llega en el tiempo requerido, limitando así la capacidad de decisión de las autoridades de salud pública para intervenir con máxima eficacia. Por lo que es necesario el incremento de recursos para infraestructura, equipos y recursos humanos para mejorar la calidad. (Sierra Amor, 2006) (Molero, Panunzio, Cruz, \& Núñez, 2010)

En la actualidad hay mayores exigencias para que los laboratorios clínicos utilicen sus recursos efectivamente y se desempeñen con calidad. (Donabedian, 1991) (Briozzo \& Perego, 2002)

El manejo de la calidad que incluye los conceptos más tradicionales del control de calidad, el aseguramiento de la calidad y la evaluación externa de la calidad que se enmarcan en la gestión de la calidad de los laboratorios clínicos. (Etcheverry, 2016) (Fundación Bioquímica Argentina, 1999)

Se precisa de manera inmediata la redefinición de la misión de la salud que hasta ahora se ha manejado en el Ecuador, primero con la seguridad, de que todos los hospitales públicos y por ende sus laboratorios clínicos cumplan con los requerimientos mínimos establecidos para su funcionamiento.

En la actualidad no todos los Laboratorios Clínicos de los hospitales del Ministerio de Salud Pública cuentan con un Sistema de Gestión de la Calidad basado en la norma ISO 9001:2015.

Los laboratorios catalogados como nivel II, no presenta un sistema de gestión de la calidad, aunque existe información y procedimientos que no están debidamente estructurados y organizados, que permitan la satisfacción del cliente, tanto interno como externo, que son los pacientes y los 
médicos de esta casa de Salud, que en un futuro no muy lejano, permitan fortalecer este Servicio, e identificando oportunidades de mejora al cliente.

El objetivo de la presente investigación es diseñar una propuesta de un Sistema de Gestión de Calidad en Laboratorios de nivel II, en las áreas de química clínica y hematología, en base a los requisitos de la norma ISO 9001:2015, en el cual se establezcan planes, indicadores de calidad, control de calidad interno a base de una encuesta, para la atención a los usuarios externos del laboratorio clínico.

\section{Metodología.}

\section{Enfoque de la investigación}

La investigación del presente trabajo de investigación se sustenta en el paradigma mixto debido a que se realiza la recolección de datos a lo que se revisa la lista de verificación de los requisitos de la ISO 9001:2015.

\section{Tipo de investigación}

La investigación que se aplicó al presente trabajo se considera de tipo descriptivo y observacional, que consistió en la recopilación de toda la información generada en las áreas de química clínica y hematología de los Laboratorios estudiados en la ciudad de Guayaquil, que generó los datos para poder diseñar la propuesta del Sistema de Gestión de Calidad en base a la Norma ISO 9001:2015 y permitió desarrollar instrumentos y técnicas que se utilizó como material de apoyo proveniente de fuentes primarias y secundarias

\section{Población.}


La población del presente estudio fueron 5 Laboratorios clínicos con categoría nivel II, de la ciudad de Guayaquil.

\section{Encuesta a pacientes}

Se realizó la encuesta de satisfacción al clientes con el propósito de conocer la atención al cliente por parte de los laboratorios clínicos estudiados, para lograr la satisfacción de los usuarios y consideren que los resultados son fiables, la encuesta consta de 17 ítems y se aplicó a 116 pacientes. Después de evaluar los resultados de la encuesta y sirvió como base para proponer planes de calidad para cumplir con las expectativas de los usuarios del servicio.

\section{Diseño de Sistema de Gestión de Calidad}

Esta propuesta se la realizó basada en los requisitos de la norma ISO 9001:2015 y básicamente consta del siguiente esquema de documentación:

Elaboración del manual de calidad: Se verificó con el check list de la norma los requisitos que cumplen, no cumplen y parcialmente cumplen en las áreas de química clínica y hematología, luego se realizó el manual de calidad con los respectivos procedimientos y registros, para cumplir con los requisitos de la norma ISO 9001:2015 y pueda servir de referencia para las demás áreas del laboratorio clínico.

Elaboración de planes estratégicos: Se realizó fichas de proceso de los procesos internos: el pre-ensayo con la descripción del proceso en un diagrama de flujo, el ensayo y post-ensayo a más del proceso en el diagrama de flujo contienen su respectivo procedimiento, se elaboró el plan de compras mismo que se encuentra en el manual de calidad en la eliminación de desechos. 
Instrumentos de Recolección de Datos

El instrumento que se utilizó en el cuestionario de la encuesta se evaluó y por tanto señala que recopila información fiable, para lo cual se aplicó el coeficiente de Kuder-Richardson (KR-20), que es un indicador de consistencia interna que toma valores entre 0 y 1 .

Estos instrumentos se sometieron a validación, por parte de especialistas en el tema, la lista de verificación de la norma ISO 9001:2015 y las encuestas.

En la presente investigación se utilizó instrumentos con ítems dicotómicos valorados, en los cuales sólo existen respuestas correctas (SI) e incorrectas (NO), es decir, que permite obtener la confiabilidad a partir de los datos obtenidos en una sola aplicación del test de los pacientes encuestados. Se procedió a agrupar numéricamente los datos y luego se realizó el cálculo con los datos, como si se tratara de datos cuantitativos y se analizó correctamente. 


\section{Resultados.}

La estructura de la documentación del sistema de calidad se definió de la siguiente manera:

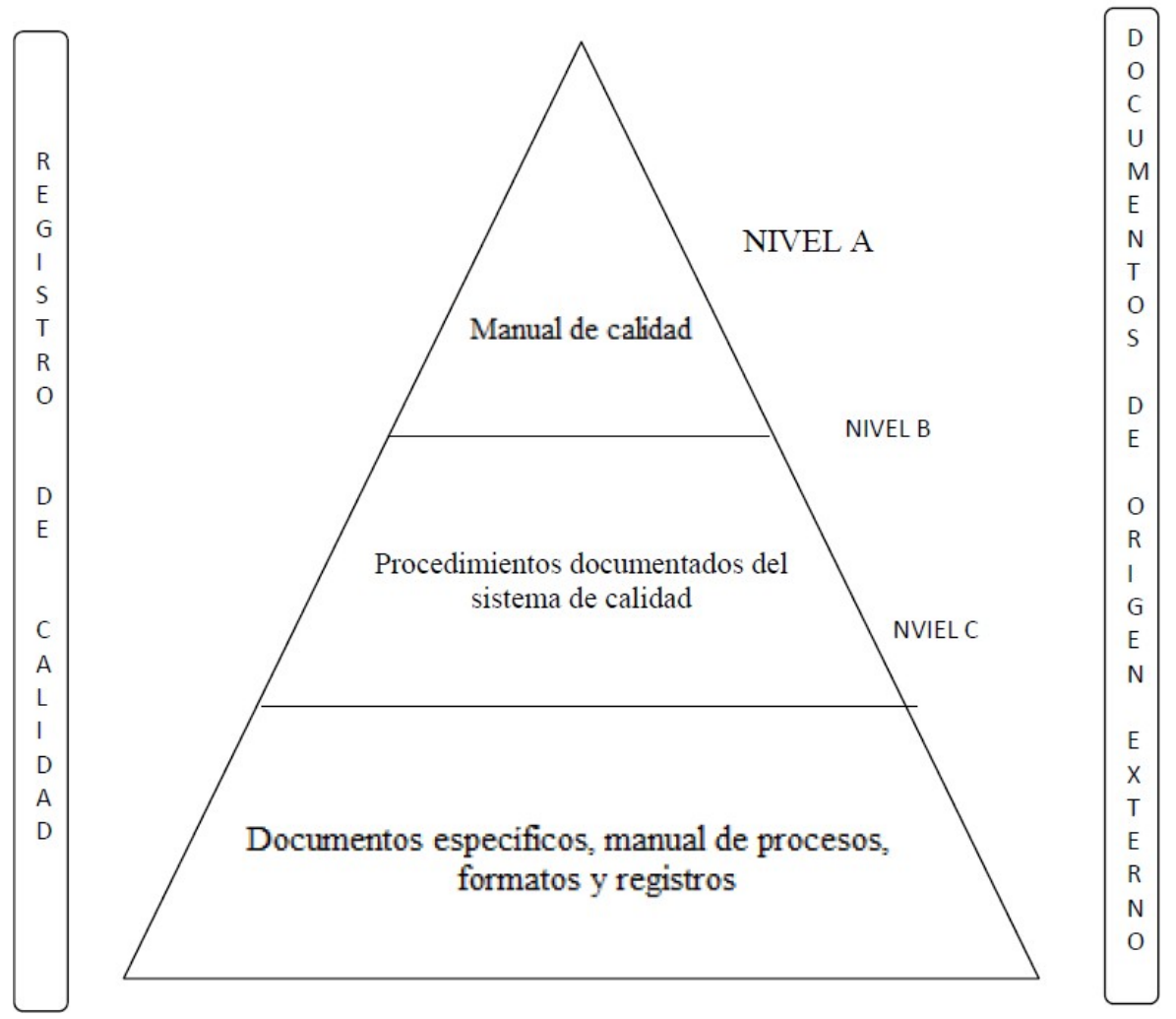

Figura $N^{\circ}$ 1.- Estructura de la documentación

Nivel A: Documento de mayor nivel en el sistema de gestión de la calidad y constituye el manual de calidad, en el que está contenida la política y objetivos de calidad y de él se derivan los siguientes niveles de la documentación.

Nivel B: Lo integran los procedimientos documentados del SGC, que aplica el personal que participa en su ejecución. 
Nivel C: Se constituye por los procedimientos de uso general, procedimientos específicos de procesos que rigen su ejecución, procedimientos operativos o de trabajo particular de casa etapa de proceso, instrucciones de trabajo que contienen en forma detallada tareas para realizar una actividad específica (instructivos, formatos, normas).

\section{Manual de calidad}

El manual de calidad de una organización, es un documento donde se especifican la misión y visión de una empresa con respecto a la calidad así como la política de la calidad y los objetivos que apuntan al cumplimiento de dicha política.

Las áreas de química clínica y hematología de los laboratorios es indispensable implementar dicho manual de calidad ya que se obtienen beneficios como satisfacción de atención a los clientes, disminución de quejas, entrega a tiempo de los resultados, se mejora el clima laboral y las relaciones humanas, creando conciencia respecto a la calidad que se debe tener, se mejora la cultura de la calidad, confianza de los clientes, resultados óptimos y confiables.

\section{Procedimientos documentados}

- Control de documentos: define las acciones de control para la elaboración y aprobación de documentos, su revisión y actualización, su identificación, almacenamientos y recolección en caso de ser obsoleto.

- Control de registros: precisa las actividades de control relacionadas con la identificación, almacenamiento, protección y recuperación. 
- Auditorías internas de calidad: especifica las responsabilidades de los participantes de la auditoría y las acciones relativas a la planificación, realización y los registros de la misma.

- Control del producto no conforme: establece las labores relacionadas con la identificación del producto no conforme y la prevención de su entrega no intencionada.

- Acciones correctivas y preventivas: describe las actividades concernientes a la revisión de las no conformidades y la identificación de sus causas, la evaluación, determinación e implementación de las acciones para evitar la ocurrencia de las mismas. En cuanto a las acciones preventivas, detalla las acciones encaminadas a la eliminación de las causas de las no conformidades potenciales para evitar su ocurrencia.

\section{Control de los registros}

La organización debe establecer un procedimiento documentado para definir los controles necesarios para la identificación, el almacenamiento, la protección, la recuperación, la retención y la disposición de los registros.

En las áreas de química clínica y hematología es importante llevar un registro ya que con esto se demuestra que ls procesos se están llevando a cabo de forma adecuada y por último se puede verificar y hacer seguimiento a los procesos.

\section{Compromiso de la dirección}

La alta dirección debe proporcionar evidencia de su compromiso con el desarrollo e implementación del sistema de gestión de calidad, así como la mejora continua de su eficacia. 
En las áreas de química clínica y hematología el compromiso de la dirección es muy importante ya que el en proceso de la calidad, el gerente es él toma las decisiones, llevando a la entidad al surgimiento o fracaso económico.

Otros documentos del sistema de gestión de la calidad

Comprende formatos tales como listados o programas. Son documentos útiles para registrar datos e información en general para su uso posterior. Este grupo de documentos se encuentran en la lista maestra de registros.

La propuesta metodológica que incluye todos los documentos del Sistema de Gestión de Calidad.

Metodología a seguir en el diseño del plan de acción del sistema de gestión de calidad.

\section{ETAPAS}

\section{ETAPA DE PLANEACIÓN}

Objetivo. Establecer los parámetros necesarios para determinar y desarrollar métodos, tareas y tiempos requeridos por la norma.

Actividades. Plan detallado de trabajo.

Plan de comunicación.

Estructura y roles. 


\section{ETAPAS DE DIAGNÓSTICO}

Objetivo. Analizar el diagnóstico del estado de las áreas de química clínica y hematología en el

HPAS, frente a los requisitos exigidos por la norma.

Actividades. Establecer un grupo de trabajo que analice la información recolectada a través de los instrumentos.

Presentación de los resultados de la encuesta.

Hacer la revisión por parte de la dirección del laboratorio clínico.

\section{ETAPA DE DISEÑO}

Objetivo. Establecer todos los elementos generales necesarios par a la elaboración del sistema documental.

Actividades. Diseñar e implementar documentos exigidos por la norma de calidad.

Organizar y controlar los diferentes procesos.

Diseñar el manual de funciones.

Realizar seguimiento y control a los procesos.

Presentar informes.

\section{ETAPA DE GESTIÓN DEL CAMBIO}

Objetivo. Determinar el impacto de los cambios y la aceptación de las personas al establecer un plan de acción. 
Actividades. Programación de charlas de sensibilización.

Sesiones de presentación del proyecto del SGC.

Talleres para entender y comprender el SGC.

Capacitaciones internas.

En los laboratorios clínicos debe responsabilizarse de la elaboración de un plan de trabajo que será necesario para llevar a cabo la implementación del sistema de gestión de calidad, donde se establezcan roles y responsabilidades de cada integrante de la organización.

Una vez que el laboratorio clínico en las áreas de química clínica y hematología haya determinado los objetivos, actividades, responsables, cronograma, recursos y mecanismo de evaluación, debe establecer los medios más efectivos y dinámicos para gestionar su debido desarrollo.

Es necesario para las áreas de química clínica y hematología que tiene por objetivo certificarse en calidad, el cumplimiento de los documentos exigidos por la norma, dando a conocer al personal la mejora continua y adquirir nuevos conocimientos que le permitan acceder a la certificación. Se incluye el plan de implementación.

\section{Conclusiones.}

Se logró ejecutar el diseño de una propuesta de un plan de implementación de la norma ISO 9001:2015 en los Laboratorios Clínicos en las áreas de química clínica y hematología, al tener un nivel de cumplimiento bajo de los requisitos de la norma en las áreas mencionadas se cuenta con un personal y directivos comprometidos es posible aplicarlo. 
Con la elaboración de los manuales de calidad, de procedimientos y procesos desarrollados en el presente trabajo se logró un incremento de un 40\% sobre el nivel de cumplimiento documental de los requisitos para la acreditación del servicio de laboratorio ante la normativa ISO 9001:2015.

\section{Bibliografía.}

Briozzo, G., \& Perego, M. (2002). El gerenciamiento del laboratorio de análisis clínicos con la visión de la calidad total. Revista del Hospital Materno Infantil Ramón Sardá, 21(1), 28-33.

Donabedian, A. (1991). La calidad de la atención médica. México: La Prensa Médica Mexicana.

Etcheverry, G. (2016). Gestión de Calidad en Laboratorios Clínicos: Implementación de Gestión Clínica. Bioanalisis, 15(2), 9-18.

Fundación Bioquímica Argentina. (1999). Manual de Acreditación de Laboratorios, MA2. Buenos Aires: Fundación Bioquímica Argentina.

Molero, T., Panunzio, A., Cruz, S., \& Núñez, M. (2010). Gestión de la calidad de atención en laboratorios clínicos de hospitales públicos en Maracaibo, Venezuela. Rev. salud pública, 12(4), 658-668.

Sierra Amor, R. (2006). El laboratorio clínico y el control de calidad. Bioquimia, 31(2), 39-40. 